\title{
Banco de dados das aves amostradas no Parque Nacional Saint-Hilaire/Lange e entorno em 2012 e 2013, Floresta Atlântica no Litoral do Paraná.
}

\author{
Database of birds sampled in the Saint-Hilaire/Lange National Park and their surroundings in \\ 2012 and 2013, Atlantic Forest of Paraná Coast.
}

Recebido: 12/06/2021 | Aceito: 26/07/2021 | Publicado: 12/08/2021 https://doi.org/10.53805/lads.v1i1.22

\author{
Luiz Augusto M. Mestre ${ }^{* 1}$
}

\section{RESUMO}

Neste estudo, apresentamos os dados de amostragens das aves registradas no Parque Nacional Saint Hilaire/Lange e em áreas de seu entorno (no município de Matinhos, Paraná). Os dados foram coletados em florestas submontana das encostas, abaixo de $200 \mathrm{~m}$ de altitude, em quatro expedições de campo (1-13 de outubro 2012, 7-17 de dezembro 2012, 19-27 de março 2013 e 18-26 de julho 2013). Foram usadas 30 redes de neblina para as capturas ( 2 grupos de 15 redes de $12 \mathrm{~m}$, malha $30 \mathrm{~cm}$ ), abertas em dois dias consecutivos em cada área (total $5400 \mathrm{~h} /$ redes). Ainda foram registradas as aves em 184 pontos de escuta (10 minutos, raio 50m) feitos próximos as áreas amostradas. Foram capturados 1666 indivíduos de 98 espécies (destes, 416 foram recapturados). Nos pontos de escuta, foram registrados 2051 indivíduos de 143 espécies. As espécies mais capturadas foram Turdus albicollis, Ramphodon naevius, Chiroxiphia caudata, Thalurania glaucopis e Tachyphonus coronatus. Nos pontos de escuta, as espécies mais frequentemente registradas foram Tangara cyanocephala, Basileuterus culicivorus, Turdus flavipes, Chiroxiphia caudata e Ramphodon naevius. A avifauna ocorrente nas áreas modificadas no entorno do Parque é influenciada pela matriz e pela estrutura local da vegetação. O presente estudo fornece dados da abundância e riqueza de aves para uma região pouco estudada e de grande diversidade biológica, permitindo avaliar a distribuição de aves e as áreas no entorno do Parque Nacional Saint Hilaire/Lange. Essas informações podem ser utilizadas em futuros estudos e ações conservacionistas na área.

Palavras-chave: Comunidade de aves; Mata Atlântica; Pontos de escuta; Redes de neblina; Unidade de Conservação.

\footnotetext{
${ }^{1}$ Universidade Federal do Paraná, LabOrnito, Setor Litoral, Matinhos, Paraná, Brasil. luiz.mestre@ufpr.br
} 


\section{ABSTRACT}

We present sampling data of bird communities registered in Saint Hilaire/Lange National Park, including surrounding areas in the municipality of Matinhos, Paraná, Brazil. The data was collected in slope and lowlands Atlantic forests, bellow 200m altitude, in four expeditions (1-13 October 2012, 7-17 December 2012, 19-27 March 2013 and 18-26 July 2013). We used 30 mist nets ( 2 groups of 15 nets of $12 \mathrm{~m}$, mesh $30 \mathrm{~cm}$ ), opened in two days in each site (total $5400 \mathrm{~h} /$ nets). We also registered the birds in 184 point counts (10 minutes, 50m). We captured 1666 individuals of 98 species (416 was recaptured). In the point counts we registered 2051 individuals of 143 species. The most captured birds species were Turdus albicollis, Ramphodon naevius, Chiroxiphia caudata, Thalurania glaucopis and Tachyphonus coronatus. In the point counts the most frequent species were Tangara cyanocephala, Basileuterus culicivorus, Turdus flavipes, Chiroxiphia caudata and Ramphodon naevius. The avifauna registered in modified sites in the surroundings of the National Park is influenced by the matriz and forest structure. The present study provides bird abundance and richness data for a little studied but highly diverse region, permitting evaluate the bird distribution near the Saint Hilaire/Lange National Park. These information may assist future conservation actions in the area.

Keywords: Atlantic Forest; Bird community; Mist-nets; Point-counts; Protected Areas.

\section{PUBLICAÇÕES PRÉVIAS}

MESTRE, L. A. M. et al. Aves do Parque Nacional Saint Hilaire/Lange e entorno. In: Alan Ripol Alves; Pós Graduação Desenvolvimento Territorial Sustentável. (Org.). Litoral do Paraná: Território e Perspectivas. 1 ed. Curitiba: Brazil Publishing, v. 4, p. 133-186, 2020.

BONI, J. et al. Aves do Parque Municipal do Tabuleiro, Matinhos, Paraná. Atualidades Ornitológicas (Online), v. 208, p. 10-17, 2019.

MESSIAS, A. et al. Papagaios como ferramentas para a Educação Ambiental. Educação Ambiental em Ação, v. 59, p. 1-64, 2017.

\section{IMPORTÂNCIA DOS DADOS}

- Fornece dados da abundância e riqueza de aves para uma região pouco estudada e de grande diversidade biológica, incluindo o Parque Nacional Saint Hilaire/Lange e seu entorno.

- Avalia a distribuição de aves no entorno do Parque Nacional Saint Hilaire/Lange.

- Permite a avaliação de áreas de interesse e impactadas no entorno do Parque Nacional Saint Hilaire/Lange. 


\section{MATERIAL E MÉTODOS}

\section{Área de Estudo}

O presente estudo foi realizado no Parque Nacional Saint-Hilaire/Lange (PNSHL), no município de Matinhos, Paraná (24⒈ $18^{\prime} 00^{\prime \prime} \mathrm{S}$ e $53^{\circ} 55^{\prime} 30^{\prime \prime}$ W). Essa Unidade de Conservação tem aproximadamente 25.000 hectares de Floresta Atlântica e foi criada em 23 de maio de 2001 (BRASIL, Lei Federal no 10.227, de 23 de maio de 2001). Situa-se na porção sul da Serra do Mar, abrangendo regiões que variam de 10 metros sobre o nível do mar até altitudes superiores a 1.400 metros (Montanhas do maciço Serra da Prata). O Parque está inserido na área-núcleo da Reserva da Biosfera da Mata Atlântica e na Área de Proteção Ambiental Estadual de Guaratuba (BLUM; RODERJAN, 2007). O Parque Nacional Saint Hilaire/Lange e seu entorno têm tipologias vegetacionais bem representadas em seus domínios, podendo ser divididas com base em seus sedimentos de origem, cada um com características estruturais e florísticas determinadas pelo clima, relevo e solos, distinguindo as formações sobre os sedimentos oceânicos (Floresta Aluvial e das Terras Baixas) e sobre sedimentos continentais (Submontana, Montana e Altomontana) (BLUM; RODERJAN, 2007). A vegetação no entorno do Parque é representada principalmente pela floresta Ombrófila Densa das Terras Baixas, incluindo também as formações pioneiras com influência marinha (restingas) e flúvio-marinha (manguezais). A vegetação secundária destes tipos vegetacionais também está presente no entorno, principalmente nas regiões mais baixas (BLUM, 2010).

Figura 2. Área de Estudo no Parque Nacional Saint Hilaire/Lange (PNSHL) e entorno, em Matinhos, Litoral do Paraná, Brasil.

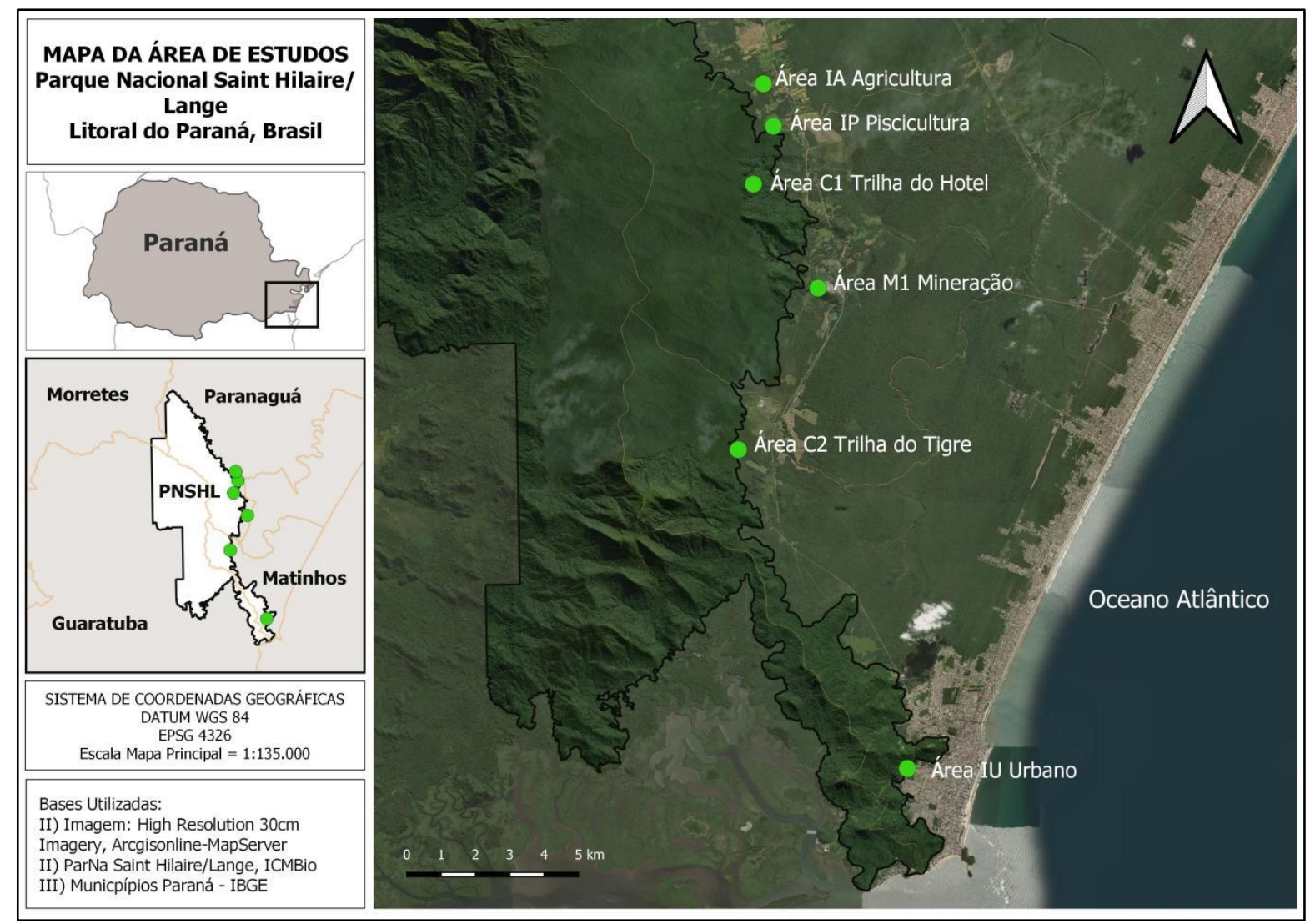




\section{Coleta de dados}

As amostragens da comunidade de aves foram realizadas com redes de neblina e pontos de escuta entre os anos de 2011 e 2013. Em florestas tropicais, os pontos de escuta tendem a ser mais efetivos amostrando aves em todos os extratos florestais, ao mesmo tempo a utilização de redes neblina oferece mais robustez ao método, por amostrar aves que não estão cantando, capturando mais espécies que voam a altura da rede (e.g. BLAKE; LOISELLE, 2001; MESTRE et al., 2020). As amostragens foram realizadas nas seguintes expedições de campo: a) 30 de agosto a 5 de setembro, e 14 a 21 de dezembro de 2011; b) de 2 a 9 de julho, 3 a 14 de outubro, e 30 de novembro a 17 de dezembro de 2012; c) 19 a 27 de março, 13 a 26 de julho de 2013. Essas amostragens foram realizadas em duas áreas em melhor estado de conservação (trilha do Tigre e próximas ao Hotel Mata Atlântica) e em áreas antropizadas (próximas de agricultura, piscicultura, mineração e também áreas próximas a cidade de Matinhos, Fig. 1).

Foram usadas 30 redes de neblina para capturar as aves (dois grupos de 15 redes de nylon preto com malha de $30 \mathrm{~cm}$ ), abertas em dois dias consecutivos em cada área. $\mathrm{O}$ método utilizando redes de neblina é importante em florestas tropicais, pois captura de forma padronizada as aves de subbosque que são as mais suscetíveis a distúrbios florestais (KARR, 1981; JOHNS, 1991). As redes de neblina foram dispostas em trilhas predefinidas e para cada amostragem foram utilizadas 30 redes de neblina $(12 \times 2.5 \mathrm{~m} ; 36 \mathrm{~mm})$ dispostas nas trilhas do Parque, incluindo áreas de pouco/nenhum uso, e áreas de uso intenso (como de visitação turística, de agricultura e/ou habitações humanas do Parque ou seu entorno. As redes de neblina foram abertas durante dois dias em cada área, desde o amanhecer (aproximadamente às $6 \mathrm{~h}$ ) e fechadas no início da tarde (aproximadamente 13h). Este protocolo objetivou a captura de uma maior proporção de espécies, aliada a replicação e a logística disponível, seguindo metodologia já aplicada em outros estudos (BARLOW; HAUGAASEN; PERES, 2002; BARLOW; PERES, 2004; CAVARZERE et al., 2013). As redes de neblina foram revisadas a cada uma hora e fechadas durante os períodos de forte chuva. Todas as aves capturadas foram identificadas com base em caracteres morfológicos descritos em guias de campo e posteriormente com fotos disponíveis na internet (VAN PERLO, 2009; NAROSKY; YZURIETA, 2010). Foram fotografadas a maioria das espécies capturadas. Quando possível, os indivíduos foram sexados e marcadas com anilhas cedidas pelo Centro Nacional de Pesquisa para Conservação de Aves Silvestres - ICMBio (Seguindo os critérios do Manual de Anilhamento de Aves Silvestre CEMAVE - IBAMA, 1994). Após esse procedimento, as aves foram soltas no mesmo local de captura.

Conjuntamente a esse método, foram realizadas amostragens em 120 pontos de contagem, registrando e gravando as aves por 10 minutos, em um raio estimado de $50 \mathrm{~m}$. Cada ponto de escuta consistiu na tomada de dados num intervalo de 10 minutos no qual foram observadas e gravadas as espécies de aves visualizadas em um raio de 50 metros (BIBBY et al., 1998; HILL; HAMER, 2004). Os pontos eram espaçados em no mínimo 150 metros, considerando-se as dificuldades em terreno escarpado e a paisagem ser composta por um mosaico de tipos de uso (BIBBY et al., 1998). As amostragens foram realizadas entre as 6:00h e 9:30h (horário de maior atividade de aves). As aves foram identificadas e quantificadas visualmente pelo uso binóculos (se possível). As identificações foram confrontadas em guias de campo e com fotos disponíveis na internet (VAN PERLO, 2009; NAROSKY; YZURIETA, 2010). Os registros sonoros foram gravados com um gravador digital (e microfone), o que assegura a correta identificação das espécies amostradas. As vocalizações das espécies de aves foram 
conferidas e comparadas com vocalizações disponíveis em CDs especializados (i.e., GONZAGA; CASTIGLIONI, 2001) ou na internet (www.wikiaves.com.br; www.xeno-canto.org). As espécise de aves capturada nas redes ou registradas nos pontos foram identificadas com base na lista do Conselho de Registros Ornitológicos (PIACENTINI et al., 2015).

\section{DESCRIÇÃO DE DADOS}

Foram capturadas com as redes de neblina 1666 indivíduos de 98 espécies (recapturadas 416 destes indivíduos). Nos pontos de escuta foram registrados 2051 indivíduos de 143 espécies de aves (Apêndice, Tabelas 1 e 2). As espécies mais capturadas com redes foram Turdus albicollis, Ramphodon naevius, Chiroxiphia caudata, Thalurania glaucopis e Tachyphonus coronatus. Nos pontos de escuta, foram registrados em maior abundância as espécies Tangara cyanocephala, Basileuterus culicivorus, Turdus flavipes, Chiroxiphia caudata e Ramphodon naevius. A avifauna ocorrente nas áreas modificadas no entorno do Parque parece ser influenciada pela matriz e pela estrutura local da vegetação. Essas informações devem ser consideradas em estudos futuros e serão incluídas no plano de manejo deste Parque Nacional.

\section{Banco de Dados}

O banco de dados (Dataset AvesPNSHL_Mestre2012_2013) é um arquivo Excel, constituído por quatro abas 1) REDES DE NEBLINA; 2) Legenda_Redes_Neblina; 3) PONTOS DE ESCUTA; 4) Legenda_Pontos_Escuta

\section{1) REDES NEBLINA, colunas:}

- Captura: Número do indivíduo que foi capturado.

- Data: Data da amostragem em campo.

- Gruporede: A e B são grupos de 15 redes separadas em ao menos 500 metros entre si.
C1 - Area Controle 1, Hotel Mata Atlântica (25.6716, -48.5988)

C2 - Area Controle 2, Trilha do Cachoeira Tigre (25.7411, -48.6032)

IP - Area Impacto Piscicultura

$(-25.6564$ -48.593)

IM - Area Impacto Mineração 25.6988, -48.5801)

IA - Area de Impacto Agricultura (-25.6453, 48.5959)

IU - Area Impacto Urbano (-25.8245, -48.5541)

-Status_1Cap2Recap: 1 Aves capturada uma vez, 2 Ave recapturada.

-Especie: Espécie de ave capturada nas redes ou registradas nos pontos com base no Conselho de Registros Ornitológicos, 2015.

- Ordem: Ordem de ave capturada nas redes ou registradas nos pontos.

- Familia: Família de ave capturada nas redes ou registradas nos pontos.

- Sexo: Sexo da ave capturada (IBAMA, 1994; VAN PERLO, 2009).

-Idade: Idade da aves caturada, A - Adulto, J Jovem, I - Indeterminado (IBAMA 1994; van Perlo, 2009).

-X e Y: coordenadas em formato UTM, todas no Fuso 22e datum $W$.

2) Legenda_Redes_Neblina: Legenda apresentada acima.

\section{3) PONTOS DE ESCUTA;}

-Ponto: Número do ponto de escuta considerado continuamente.

- Data: Data da amostragem em campo.

- Hora: Hora da amostragem do ponto.

-Area:

C1 - Area Controle 1, Hotel Mata Atlântica (25.6716, -48.5988)

C2 - Area Controle 2, Trilha do Cachoeira Tigre (-25.7411, -48.6032) IP - Area Impacto Piscicultura $(-25.6564$ $-48.593)$

-Area: 
IM - Area Impacto Mineração (-25.6988, 48.5801)

IA - Area de Impacto Agricultura (-25.6453, 48.5959)

IU - Area Impacto Urbano (-25.8245, 48.5541)

-PontoArea: Número de pontos de escuta realizados na Area.

-Ano e expedicao: Ano de realização e Expedição feita $A=$ primeira, $B=$ Segunda,$C=$ Terceira em determinada Area.
- Especie: Espécie de ave capturada nas redes ou registradas nos pontos com base no Conselho de Registros Ornitológicos (PIACENTINI et al., 2015).

- Ordem: Ordem de ave capturada nas redes ou registradas nos pontos.

- Familia: Família de ave capturada nas redes ou registradas nos pontos. $\bullet \mathrm{X}$ e $\mathrm{Y}$ : coordenadas em formato UTM, todas no Fuso 22e datum W.

4) Legenda_Pontos_Escuta: Legenda apresentada acima.

\section{ARQUIVOS SUPLEMENTARES}

Banco de dados: AvesPNSHL_Mestre2012_2013.

\section{AGRADECIMENTOS}

Agradeço ao ICMBio (DIBIO) pelo apoio logístico e financeiro. Agradeço a importante participação nos trabalhos de campo de Ricardo Krul, Luciana Festti, Rodrigo F. Torres, Rogério Florenzano Jr, Beatriz Gomes, Luiz Faraco e os diversos voluntários do projeto.

\section{REFERÊNCIAS}

BARLOW, J.; HAUGAASEN, T.; PERES, C. A. Effects of ground fires on understorey bird assemblages in Amazonian Forests. Biological Conservation, 105, 157-169, 2002. DOI: https://doi.org/10.1016/S00063207(01)00177-X.

BARLOW, J.; PERES, C. A. Ecological responses to El Nino-induced surface fires in central Amazonia: management implications for flammable tropical forests. Philosophical Transactions of the Royal Society of London B. 359, 367-380, 2004. DOI: https://doi.org/10.1098/rstb.2003.1423.

BIBBY, C.; JONES, M.; MARSDEN, S. Expedition field techniques: bird surveys. London: Royal Geographical Society, London, UK, 1998. Available at http://www.bio-nica.info/ALAS/pdf2.pdf

BLAKE, J. G.; LOISELLE, B. A. Bird assemblages in second-growth and old-growth forests, Costa Rica: perspectives from mist nets and point counts. Auk. 118, 304-326, 2001.

BLUM, C. T. O componente epifítico vascular e herbáceo terrícola da Floresta Ombrófila Densa ao longo de um gradiente altitudinal na Serra da Prata, Paraná. Tese Doutorado. Universidade Federal do Paraná. 197p, 2010.

BLUM, C. T.; RODERJAN, C. V. Espécies indicadoras em um gradiente da Floresta Ombrófila Densa na Serra da Prata, Paraná, Brasil. Revista Brasileira de Biociências, Porto Alegre, 5: 873-875, 2007.

CAVARZERE, V. et al. Evaluation of methodological protocols using point counts and mist nets: a case study in southeastern Brazil. Papéis Avulsos Zoologia, 53 (26), 345-356, 2013. DOI: https://doi.org/10.1590/S003110492013002600001. 
GONZAGA, L. P.; CASTIGLIONI, G. Aves das montanhas do Sudeste do Brasil. Manaus: Sonopress. CD Áudio, 2001.

HILL, J. K.; HAMER, K. C. Determining impacts of habitat modification on diversity of tropical forest fauna: the importance of spatial scale. Journal of Applied Ecology. 41, 744- 754, 2004.

IBAMA. Manual de anilhamento de aves silvestres 2a edição. Instituto Brasileiro do Meio Ambiente e dos Recursos Naturais Renováveis, Brasília, DF, 1994.

JOHNS, A. D. Responses of Amazonian rain-forest birds to habitat modification. Journal of Tropical Ecology. 7, 417-437, 1991.

KARR, J. R. Surveying birds in the tropics. Studies in Avian Biology 6, 548-553, 1981.

MESTRE, L. A. M. et al. Impacts of selective logging on avian phylogenetic and functional diversity in the Amazon. Animal Conservation, v. 23, p. 725-740, 2020. DOI: https://doi.org/10.1111/acv.12592.

NAROSKY, T.; YZURIETA, D. Aves de Argentina y Uruguay - Birds of Argentina \& Uruguay: Guía de Identificación - A Field Guide. 16a ed. Vázquez Mazzini Editores, Buenos Aires, Argentina, 2010.

PIACENTINI, V. et al. Annotated checklist of the birds of Brazil by the Brazilian Ornithological Records Committee. Brazilian Journal of Ornithology, 23(2), 90-298, 2015. Retrieved from http://www.revbrasilornitol.com.br/BJO/article/view/1263. DOI: https://doi.org/10.1007/BF03544294.

VAN PERLO, B. A field guide to the Birds of Brazil. Oxford University Press, New York, EUA, 2009.

\section{APÊNDICE}

Tabela 1. Listagem das aves capturadas nas redes de neblina em 2012 e 2013 no Parque Nacional Saint-Hilaire/Lange, Matinhos, Paraná. Espécies em ordem alfabética conforme identificação CBRO 2017. C1 - Área Controle 1, Região Hotel Mata Atlântica. C2 Área Controle 2 - Região da Trilha da Cachoeira do Tigre, IA - Área Impacto Agricultura, IM - Área Impacto Mineração, IP - Área Impacto Piscicultura, IU - Área de Impacto Urbana.

\begin{tabular}{llllllll}
\hline ESPÉCIES & C1 & C2 & IA & IM & IP & IU & Total \\
\hline Amazilia versicolor & 0 & 7 & 6 & 8 & 1 & 8 & 30 \\
Aphantochroa cirrochloris & 0 & 0 & 3 & 0 & 0 & 2 & 5 \\
Arremon semitorquatus & 1 & 0 & 0 & 0 & 0 & 0 & 1 \\
Attila rufus & 2 & 0 & 1 & 0 & 7 & 3 & 13 \\
Automolus leucophthalmus & 0 & 5 & 2 & 3 & 1 & 10 & 21 \\
Baryphthengus ruficapillus & 2 & 0 & 0 & 0 & 0 & 0 & 2 \\
Basileuterus culicivorus & 16 & 0 & 13 & 10 & 27 & 32 & 98 \\
Cantorchilus longirostris & 0 & 0 & 1 & 0 & 1 & 3 & 5 \\
Carpornis cucullata & 0 & 1 & 0 & 0 & 0 & 0 & 1 \\
Celeus flavescens & 0 & 1 & 1 & 0 & 1 & 0 & 3 \\
Chamaeza campanisona & 0 & 0 & 0 & 0 & 0 & 1 & 1 \\
Chiroxiphia caudata & 17 & 36 & 28 & 10 & 17 & 35 & 143 \\
Chloroceryle aenea & 0 & 0 & 0 & 0 & 0 & 1 & 1 \\
Chloroceryle americana & 0 & 0 & 0 & 0 & 1 & 0 & 1 \\
Cichlocolaptes leucophrus & 0 & 0 & 0 & 0 & 1 & 0 & 1 \\
Clytolaema rubricauda & 4 & 2 & 8 & 0 & 1 & 9 & 24 \\
Coereba flaveola & 2 & 1 & 3 & 0 & 3 & 4 & 13 \\
Conopophaga lineata & 0 & 0 & 1 & 0 & 0 & 0 & 1 \\
Conopophaga melanops & 2 & 2 & 7 & 6 & 5 & 10 & 32 \\
Dendrocincla turdina & 12 & 7 & 24 & 8 & 2 & 2 & 55 \\
Dendrocolaptes platyrostris & 0 & 0 & 0 & 1 & 1 & 0 & 2 \\
\hline Pat & & & & & &
\end{tabular}




\begin{tabular}{|c|c|c|c|c|c|c|c|}
\hline Drymophila squamata & 3 & 8 & 9 & 4 & 6 & 9 & 39 \\
\hline Dysithamnus mentalis & 0 & 1 & 0 & 4 & 0 & 3 & 8 \\
\hline Dysithamnus stictothorax & 0 & 0 & 0 & 0 & 0 & 3 & 3 \\
\hline Eleoscytalopus indigoticus & 4 & 0 & 0 & 0 & 2 & 0 & 6 \\
\hline Euphonia pectoralis & 2 & 13 & 0 & 0 & 2 & 0 & 17 \\
\hline Euphonia violacea & 0 & 0 & 1 & 1 & 0 & 2 & 4 \\
\hline Florisuga fusca & 0 & 0 & 0 & 1 & 0 & 0 & 1 \\
\hline Formicarius colma & 1 & 1 & 3 & 0 & 2 & 0 & 7 \\
\hline Geothlypis aequinoctialis & 0 & 0 & 1 & 0 & 5 & 0 & 6 \\
\hline Geotrygon montana & 6 & 1 & 1 & 1 & 2 & 0 & 11 \\
\hline Habia rubica & 9 & 6 & 6 & 4 & 1 & 21 & 47 \\
\hline Haplospiza unicolor & 1 & 0 & 1 & 0 & 0 & 6 & 8 \\
\hline Hemithraupis ruficapilla & 0 & 0 & 0 & 1 & 0 & 0 & 1 \\
\hline Hemitriccus orbitatus & 2 & 2 & 0 & 7 & 3 & 1 & 15 \\
\hline Hydropsalis albicollis & 0 & 0 & 1 & 0 & 0 & 0 & 1 \\
\hline Hylophilus poicilotis & 0 & 0 & 0 & 0 & 1 & 0 & 1 \\
\hline Ilicura militaris & 1 & 1 & 1 & 2 & 1 & 3 & 9 \\
\hline Lanio melanops & 14 & 18 & 21 & 9 & 5 & 16 & 83 \\
\hline Lathrotriccus euleri & 0 & 2 & 3 & 8 & 5 & 12 & 30 \\
\hline Legatus leucophaius & 0 & 0 & 0 & 0 & 0 & 1 & 1 \\
\hline Leptopogon amaurocephalus & 14 & 2 & 6 & 10 & 5 & 4 & 41 \\
\hline Leptotila rufaxilla & 0 & 0 & 1 & 0 & 1 & 0 & 2 \\
\hline Malacoptila striata & 0 & 2 & 0 & 0 & 1 & 0 & 3 \\
\hline Manacus manacus & 0 & 1 & 18 & 1 & 13 & 32 & 65 \\
\hline Mionectes rufiventris & 9 & 13 & 15 & 7 & 7 & 16 & 67 \\
\hline Myiobius barbatus & 3 & 10 & 2 & 6 & 4 & 6 & 31 \\
\hline Myiornis auricularis & 0 & 0 & 0 & 0 & 0 & 3 & 3 \\
\hline Myrmeciza squamosa & 3 & 2 & 1 & 4 & 6 & 6 & 22 \\
\hline Myrmotherula gularis & 5 & 0 & 0 & 0 & 1 & 2 & 8 \\
\hline Myrmotherula unicolor & 3 & 4 & 7 & 6 & 4 & 2 & 26 \\
\hline Onychorhynchus swainsoni & 0 & 0 & 0 & 0 & 2 & 1 & 3 \\
\hline Oxyruncus cristatus & 0 & 0 & 1 & 0 & 0 & 0 & 1 \\
\hline Pachyramphus polychopterus & 0 & 0 & 1 & 0 & 0 & 0 & 1 \\
\hline Phaeothlypis rivularis & 1 & 6 & 6 & 0 & 11 & 1 & 25 \\
\hline Phaethornis squalidus & 11 & 1 & 6 & 3 & 5 & 7 & 33 \\
\hline Philydor atricapillus & 6 & 4 & 13 & 7 & 6 & 12 & 48 \\
\hline Philydor lichtensteini & 1 & 0 & 2 & 1 & 2 & 3 & 9 \\
\hline Phylloscartes oustaleti & 0 & 5 & 0 & 0 & 1 & 0 & 6 \\
\hline Piaya cayana & 1 & 0 & 0 & 0 & 0 & 0 & 1 \\
\hline Picumnus temminckii & 2 & 1 & 4 & 1 & 3 & 11 & 22 \\
\hline Platyrinchus leucoryphus & 0 & 2 & 0 & 0 & 1 & 0 & 3 \\
\hline Platyrinchus mystaceus & 9 & 6 & 7 & 0 & 5 & 0 & 27 \\
\hline Pyriglena leucoptera & 8 & 4 & 5 & 5 & 3 & 8 & 33 \\
\hline Ramphocelus bresilius & 0 & 0 & 1 & 0 & 0 & 0 & 1 \\
\hline Ramphodon naevius & 14 & 32 & 26 & 33 & 20 & 25 & 150 \\
\hline Rupornis magnirostris & 0 & 0 & 0 & 1 & 0 & 0 & 1 \\
\hline Schiffornis virescens & 6 & 9 & 2 & 3 & 9 & 10 & 39 \\
\hline
\end{tabular}




\begin{tabular}{llllllll}
\hline Selenidera maculirostris & 0 & 0 & 0 & 6 & 0 & 0 & 6 \\
Sporophila caerulescens & 0 & 0 & 2 & 0 & 0 & 2 & 4 \\
Sporophila falcirostris & 0 & 0 & 0 & 1 & 0 & 0 & 1 \\
Sporophila frontalis & 0 & 0 & 0 & 0 & 0 & 1 & 1 \\
Sporophila melanogaster & 0 & 0 & 0 & 0 & 0 & 1 & 1 \\
Sporophila sp & 0 & 0 & 0 & 0 & 2 & 0 & 2 \\
Stephanoxis lalandi & 1 & 0 & 0 & 0 & 0 & 0 & 1 \\
Synallaxis ruficapilla & 3 & 0 & 4 & 0 & 3 & 4 & 14 \\
Tachyphonus coronatus & 12 & 14 & 21 & 19 & 8 & 34 & 108 \\
Tangara cyanocephala & 3 & 1 & 1 & 1 & 0 & 0 & 6 \\
Tangara cyanoptera & 3 & 0 & 1 & 1 & 1 & 0 & 6 \\
Tangara seledon & 3 & 0 & 0 & 0 & 0 & 0 & 3 \\
Tersina viridis & 1 & 0 & 0 & 0 & 0 & 0 & 1 \\
Thalurania glaucopis & 12 & 31 & 46 & 11 & 13 & 15 & 128 \\
Thamnophilus caerulescens & 0 & 0 & 1 & 0 & 2 & 0 & 3 \\
Tolmomyias sulphurescens & 0 & 0 & 2 & 2 & 0 & 0 & 4 \\
Troglodytes musculus & 1 & 0 & 1 & 0 & 1 & 0 & 3 \\
Trogon rufus & 1 & 0 & 0 & 0 & 0 & 0 & 1 \\
Trogon viridis & 0 & 1 & 0 & 0 & 1 & 0 & 2 \\
Turdus albicollis & 38 & 51 & 30 & 39 & 10 & 33 & 201 \\
Turdus amaurochalinus & 0 & 0 & 0 & 0 & 2 & 0 & 2 \\
Turdus flavipes & 5 & 9 & 5 & 12 & 0 & 1 & 32 \\
Turdus rufiventris & 0 & 0 & 5 & 0 & 3 & 7 & 15 \\
Turdus subalaris & 0 & 0 & 0 & 0 & 0 & 1 & 1 \\
Tyrannus melancholicus & 0 & 0 & 0 & 0 & 1 & 0 & 1 \\
Veniliornis spilogaster & 0 & 0 & 0 & 0 & 1 & 0 & 1 \\
Vireo olivaceus & 0 & 0 & 1 & 0 & 4 & 2 & 7 \\
Xenops minutus & 1 & 6 & 6 & 2 & 4 & 7 & 26 \\
Xiphocolaptes albicollis & 0 & 1 & 2 & 1 & 0 & 0 & 4 \\
Xiphorhynchus fuscus & 5 & 11 & 21 & 9 & 8 & 21 & 75 \\
Zonotrichia capensis & 0 & 0 & 0 & 0 & 2 & 0 & 2 \\
\hline Total & $\mathbf{2 8 6}$ & $\mathbf{3 4 4}$ & $\mathbf{4 1 9}$ & $\mathbf{2 8 0}$ & $\mathbf{2 8 0}$ & $\mathbf{4 7 5}$ & $\mathbf{2 0 8 4}$ \\
\hline & & & & & & & \\
& & 0 & &
\end{tabular}

Tabela 2 - Listagem das aves registradas em pontos de escuta em 2012 e 2013 no Parque Nacional Saint-Hilaire/Lange, Matinhos, Paraná. Espécies em ordem alfabética conforme identificação CBRO, 2015. C1 - Área Controle 1, Região Hotel Mata Atlântica. C2 Área Controle 2 - Região da Trilha da Cachoeira do Tigre, IA - Área Impacto Agricultura, IM - Área Impacto Mineração, IP - Área Impacto Piscicultura, IU - Área de Impacto Urbana.

\begin{tabular}{llllllll}
\hline ESPÉCIES & C1 & C2 & IA & IM & IP & IU & Total \\
\hline Aramides saracura & 0 & 0 & 4 & 0 & 2 & 2 & 8 \\
Attila rufus & 2 & 2 & 1 & 2 & 2 & 1 & 10 \\
Automolus leucophthalmus & 2 & 2 & 0 & 1 & 0 & 3 & 8 \\
Basileuterus culicivorus & 11 & 9 & 20 & 29 & 28 & 29 & 126 \\
Brotogeris tirica & 0 & 2 & 6 & 0 & 0 & 0 & 8 \\
Cacicus haemorrhous & 0 & 6 & 0 & 2 & 2 & 3 & 13 \\
Camptostoma obsoletum & 0 & 0 & 1 & 1 & 0 & 0 & 2 \\
Cantorchilus longirostris & 0 & 0 & 3 & 0 & 7 & 0 & 10 \\
Carpornis cucullata & 0 & 0 & 1 & 0 & 0 & 1 & 2 \\
\hline
\end{tabular}




\begin{tabular}{|c|c|c|c|c|c|c|c|}
\hline Celeus flavescens & 0 & 1 & 0 & 2 & 2 & 3 & 8 \\
\hline Chamaeza campanisona & 1 & 0 & 0 & 0 & 0 & 1 & 2 \\
\hline Chiroxiphia caudata & 3 & 29 & 0 & 11 & 1 & 21 & 65 \\
\hline Cichlocolaptes leucophrus & 0 & 0 & 0 & 1 & 0 & 0 & 1 \\
\hline Coereba flaveola & 7 & 2 & 5 & 7 & 8 & 26 & 55 \\
\hline Colonia colonus & 0 & 0 & 1 & 0 & 1 & 0 & 2 \\
\hline Columbina talpacoti & 0 & 0 & 0 & 0 & 2 & 0 & 2 \\
\hline Conopophaga melanops & 0 & 1 & 0 & 3 & 0 & 1 & 5 \\
\hline Crypturellus noctivagus & 0 & 7 & 0 & 1 & 0 & 1 & 9 \\
\hline Cyanocorax caeruleus & 0 & 0 & 6 & 6 & 0 & 2 & 14 \\
\hline Dacnis cayana & 0 & 0 & 0 & 2 & 1 & 0 & 3 \\
\hline Drymophila squamata & 0 & 4 & 5 & 2 & 0 & 1 & 12 \\
\hline Dysithamnus mentalis & 1 & 1 & 2 & 0 & 0 & 1 & 5 \\
\hline Eleoscytalopus indigoticus & 0 & 2 & 2 & 0 & 1 & 1 & 6 \\
\hline Empidonomus varius & 0 & 0 & 0 & 1 & 5 & 0 & 6 \\
\hline Eupetomena macroura & 0 & 1 & 0 & 0 & 0 & 1 & 2 \\
\hline Euphonia pectoralis & 6 & 3 & 1 & 5 & 1 & 2 & 18 \\
\hline Formicarius colma & 3 & 5 & 4 & 0 & 0 & 0 & 12 \\
\hline Geothlypis aequinoctialis & 0 & 0 & 0 & 0 & 2 & 0 & 2 \\
\hline Geotrygon montana & 1 & 0 & 0 & 0 & 0 & 0 & 1 \\
\hline Habia rubica & 0 & 4 & 2 & 0 & 0 & 0 & 6 \\
\hline Haplospiza unicolor & 0 & 0 & 0 & 0 & 0 & 1 & 1 \\
\hline Hemithraupis ruficapilla & 7 & 1 & 1 & 3 & 2 & 0 & 14 \\
\hline Hemitriccus orbitatus & 1 & 1 & 0 & 0 & 1 & 0 & 3 \\
\hline Herpsilochmus rufimarginatus & 0 & 2 & 9 & 0 & 6 & 2 & 19 \\
\hline Hylophilus poicilotis & 0 & 0 & 0 & 0 & 2 & 1 & 3 \\
\hline Hypoedaleus guttatus & 0 & 2 & 0 & 1 & 0 & 0 & 3 \\
\hline Lanio melanops & 4 & 0 & 0 & 4 & 0 & 10 & 18 \\
\hline Lathrotriccus euleri & 1 & 2 & 1 & 1 & 5 & 11 & 21 \\
\hline Legatus leucophaius & 0 & 0 & 0 & 0 & 0 & 2 & 2 \\
\hline Leptopogon amaurocephalus & 0 & 0 & 1 & 0 & 1 & 1 & 3 \\
\hline Leptotila rufaxilla & 0 & 0 & 0 & 0 & 5 & 0 & 5 \\
\hline Manacus manacus & 0 & 4 & 4 & 0 & 0 & 0 & 8 \\
\hline Megarynchus pitangua & 0 & 0 & 4 & 0 & 1 & 0 & 5 \\
\hline Merulaxis ater & 0 & 1 & 0 & 0 & 0 & 0 & 1 \\
\hline Mionectes rufiventris & 0 & 1 & 0 & 1 & 0 & 0 & 2 \\
\hline Myiobius barbatus & 1 & 0 & 0 & 1 & 0 & 0 & 2 \\
\hline Myiodynastes maculatus & 2 & 3 & 0 & 1 & 2 & 5 & 13 \\
\hline Myiopagis caniceps & 0 & 0 & 0 & 0 & 2 & 0 & 2 \\
\hline Myiornis auricularis & 0 & 1 & 1 & 0 & 3 & 3 & 8 \\
\hline Myiozetetes similis & 0 & 0 & 5 & 0 & 2 & 2 & 9 \\
\hline Myrmeciza squamosa & 2 & 10 & 3 & 5 & 0 & 6 & 26 \\
\hline Myrmotherula gularis & 2 & 0 & 0 & 0 & 0 & 0 & 2 \\
\hline Myrmotherula unicolor & 0 & 0 & 0 & 2 & 0 & 0 & 2 \\
\hline Orthogonys chloricterus & 0 & 4 & 0 & 0 & 0 & 0 & 4 \\
\hline Oxyruncus cristatus & 0 & 0 & 0 & 0 & 1 & 0 & 1 \\
\hline Parula pitiayumi & 0 & 0 & 1 & 11 & 2 & 1 & 15 \\
\hline
\end{tabular}




\begin{tabular}{|c|c|c|c|c|c|c|c|}
\hline Patagioenas cayennensis & 0 & 1 & 0 & 3 & 0 & 1 & 5 \\
\hline Patagioenas picazuro & 0 & 0 & 0 & 0 & 1 & 0 & 1 \\
\hline Penelope obscura & 0 & 0 & 1 & 0 & 0 & 0 & 1 \\
\hline Phaeothlypis rivularis & 1 & 4 & 7 & 1 & 7 & 0 & 20 \\
\hline Phaethornis squalidus & 3 & 2 & 2 & 0 & 1 & 3 & 11 \\
\hline Philydor rufum & 0 & 1 & 1 & 1 & 0 & 1 & 4 \\
\hline Phylloscartes sp. & 0 & 0 & 0 & 1 & 1 & 1 & 3 \\
\hline Piaya cayana & 0 & 0 & 2 & 0 & 1 & 0 & 3 \\
\hline Piculus flavigula & 0 & 1 & 1 & 0 & 0 & 0 & 2 \\
\hline Picumnus temminckii & 4 & 8 & 9 & 7 & 8 & 5 & 41 \\
\hline Pionus maximiliani & 0 & 0 & 0 & 4 & 0 & 0 & 4 \\
\hline Pitangus sulphuratus & 0 & 1 & 6 & 0 & 5 & 4 & 16 \\
\hline Platyrinchus mystaceus & 0 & 0 & 1 & 1 & 0 & 0 & 2 \\
\hline Pteroglossus bailloni & 0 & 0 & 0 & 4 & 0 & 0 & 4 \\
\hline Pyriglena leucoptera & 3 & 4 & 2 & 0 & 0 & 1 & 10 \\
\hline Ramphastos dicolorus & 0 & 0 & 0 & 4 & 2 & 0 & 6 \\
\hline Ramphodon naevius & 10 & 16 & 16 & 6 & 7 & 10 & 65 \\
\hline Rupornis magnirostris & 0 & 0 & 2 & 0 & 4 & 1 & 7 \\
\hline Saltator similis & 0 & 0 & 0 & 0 & 1 & 0 & 1 \\
\hline Schiffornis virescens & 0 & 6 & 3 & 2 & 1 & 4 & 16 \\
\hline Sclerurus scansor & 1 & 0 & 0 & 0 & 0 & 0 & 1 \\
\hline Selenidera maculirostris & 2 & 0 & 0 & 1 & 0 & 0 & 3 \\
\hline Sicalis flaveola & 0 & 0 & 0 & 2 & 0 & 0 & 2 \\
\hline Sirystes sibilator & 0 & 0 & 0 & 0 & 5 & 0 & 5 \\
\hline Sittasomus griseicapillus & 1 & 0 & 0 & 0 & 1 & 1 & 3 \\
\hline Synallaxis ruficapilla & 0 & 0 & 3 & 0 & 0 & 2 & 5 \\
\hline Synallaxis spixi & 0 & 0 & 0 & 0 & 2 & 1 & 3 \\
\hline Tachyphonus coronatus & 3 & 2 & 7 & 8 & 9 & 7 & 36 \\
\hline Tangara cyanocephala & 19 & 24 & 30 & 29 & 22 & 28 & 152 \\
\hline Tangara sayaca & 3 & 2 & 20 & 9 & 10 & 6 & 50 \\
\hline Tangara seledon & 0 & 13 & 6 & 13 & 4 & 14 & 50 \\
\hline Thalurania glaucopis & 0 & 0 & 2 & 2 & 2 & 3 & 9 \\
\hline Thamnophilus caerulescens & 0 & 0 & 1 & 1 & 1 & 0 & 3 \\
\hline Tinamus solitarius & 2 & 1 & 0 & 0 & 0 & 0 & 3 \\
\hline Tityra cayana & 0 & 0 & 0 & 1 & 0 & 3 & 4 \\
\hline Tolmomyias sulphurescens & 3 & 3 & 4 & 3 & 3 & 3 & 19 \\
\hline Troglodytes musculus & 0 & 0 & 3 & 1 & 5 & 3 & 12 \\
\hline Trogon rufus & 2 & 0 & 0 & 1 & 0 & 0 & 3 \\
\hline Trogon viridis & 0 & 4 & 2 & 3 & 0 & 2 & 11 \\
\hline Turdus albicollis & 6 & 7 & 0 & 7 & 0 & 4 & 24 \\
\hline Turdus amaurochalinus & 3 & 1 & 1 & 1 & 0 & 0 & 6 \\
\hline Turdus flavipes & 26 & 20 & 11 & 16 & 2 & 15 & 90 \\
\hline Turdus flavipes & 0 & 1 & 1 & 1 & 0 & 0 & 3 \\
\hline Turdus rufiventris & 6 & 4 & 1 & 9 & 1 & 3 & 24 \\
\hline Tyrannus melancholicus & 0 & 1 & 1 & 2 & 6 & 4 & 14 \\
\hline Veniliornis spilogaster & 0 & 0 & 2 & 1 & 1 & 3 & 7 \\
\hline Vireo olivaceus & 11 & 3 & 1 & 12 & 5 & 16 & 48 \\
\hline
\end{tabular}




\begin{tabular}{llllllll}
\hline Volatinia jacarina & 0 & 0 & 0 & 0 & 1 & 0 & 1 \\
Xenops minutus & 0 & 0 & 1 & 0 & 1 & 0 & 2 \\
Xiphorhynchus fuscus & 12 & 8 & 2 & 1 & 3 & 3 & 29 \\
Zonotrichia capensis & 0 & 0 & 0 & 1 & 7 & 0 & 8 \\
\hline Total & $\mathbf{1 7 8}$ & $\mathbf{2 5 1}$ & $\mathbf{2 4 6}$ & $\mathbf{2 6 5}$ & $\mathbf{2 3 0}$ & $\mathbf{2 9 7}$ & $\mathbf{1 4 6 7}$ \\
\hline
\end{tabular}

